\title{
Exploratory data analysis and clustering of multivariate spatial hydrogeological data by means of GEO3DSOM, a variant of Kohonen's Self-Organizing Map
}

\author{
L. Peeters ${ }^{1}$, F. Bação ${ }^{2}$, V. Lobo ${ }^{2,3}$, and A. Dassargues ${ }^{1,4}$ \\ ${ }^{1}$ Applied Geology and Mineralogy, KULeuven, Belgium \\ ${ }^{2}$ Instituto Superior de Estatística e Gestão de Informação, Universidade Nova de Lisboa, Campus de Campolide, Lisboa, \\ Portugal \\ ${ }^{3}$ Portuguese Naval Academy, Almada, Portugal \\ ${ }^{4}$ Hydrogeology \& Environmental Geology, ArGEnCo, University of Liege, Belgium
}

Received: 17 May 2006 - Published in Hydrol. Earth Syst. Sci. Discuss.: 11 July 2006

Revised: 16 March 2007 - Accepted: 24 April 2007 - Published: 11 May 2007

\begin{abstract}
The use of unsupervised artificial neural network techniques like the self-organizing map (SOM) algorithm has proven to be a useful tool in exploratory data analysis and clustering of multivariate data sets. In this study a variant of the SOM-algorithm is proposed, the GEO3DSOM, capable of explicitly incorporating three-dimensional spatial knowledge into the algorithm. The performance of the GEO3DSOM is compared to the performance of the standard SOM in analyzing an artificial data set and a hydrochemical data set. The hydrochemical data set consists of 131 groundwater samples collected in two detritic, phreatic, Cenozoic aquifers in Central Belgium. Both techniques succeed very well in providing more insight in the groundwater quality data set, visualizing the relationships between variables, highlighting the main differences between groups of samples and pointing out anomalous wells and well screens. The GEO3DSOM however has the advantage to provide an increased resolution while still maintaining a good generalization of the data set.
\end{abstract}

\section{Introduction}

Regional monitoring of groundwater quality often yields large multidimensional data sets in which each sampling location is characterized by its geographic coordinates, longitude, latitude and height. Exploratory data analysis (EDA) and clustering can help in summarizing available data, extracting useful information and formulating hypothesis for further research.

Correspondence to: L. Peeters

(luk.peeters@geo.kuleuven.be)
Traditionally multivariate techniques like principal component analysis (PCA) and factor analysis (FA) are used in the process of exploratory data analysis and clustering (e.g. Güler et al., 2002; Lambrakis et al., 2004; Love et al., 2004). Both PCA and FA are based on linear combinations of the original variables in order to reduce the dimensionality of the data set (Davis, 1986).

Recently, artificial neural network techniques, such as Kohonen's Self-Organizing Map (SOM), have also been used in EDA. The Self-Organizing Map may be used to project multidimensional data onto a two dimensional grid in a topology preserving way, capturing complex, non-linear relationships between variables (Kohonen, 1995).

Besides applications of the algorithm in financial, medical, chemical and biological research (an overview is presented in Kaski, 1997), SOM's are also used in remote sensing (Richardson et al., 2003; Mercier et al., 2006), geophysics (Poulton et al., 1992; Ozerdem et al., 2006), geochemistry (Lacassie et al., 2004; Penn, 2005) and reservoir characterization (Chang et al., 2002). Applications of the SOMalgorithm in hydrogeological research can be found in Hong and Rosen (2001) where technique is applied to diagnose the effect of storm water infiltration on groundwater quality variables and to capture the complex nonlinear relationships between groundwater quality variables. Sanchez-Martos et al. (2002) used SOM in the classification of a hydrochemical data set from a detritic aquifer in a semi-arid region, into distinct classes of different chemical composition. Lischeid (2003) applied the self-organizing map algorithm to an intensively monitored watershed to investigate spatial and temporal trends in water quality data.

Openshaw and Turton (1996) was among the first to apply self-organizing maps to the exploratory data analysis and

Published by Copernicus GmbH on behalf of the European Geosciences Union. 
clustering of geospatial data and numerous applications of the technique in geospatial data analysis have proven to be successful (Takatsuka, 2001; Skupin and Hagelman, 2003; Koua et al., 2006). However, the inclusion of spatial ordering in the clustering or classification algorithm remains an important issue, especially in hydrogeochemical research. The chemical composition of groundwater at a certain location can be thought of as the result of geochemical processes combined with groundwater flow related phenomena as mixing, advection and diffusion. In aquifers these processes seldom result in abrupt changes in hydrochemistry, but rather show a gradual change. Based on the premise that samples located close together are more likely to be related to each other than samples with a large distance between them, the incorporation of geographical coordinates in the EDA-algorithm provides a way of accounting for the spatial correlation in the exploratory data analysis. Bação et al. (2005a) discusses this topic in relation to the SOM-algorithm and compares the standard SOM-analysis in which the geographic coordinates are considered as any other variable to the GEOSOM, a modified version of the SOM, designed to explicitly incorporate spatial information. Application of the GEOSOM on two artificial data sets and a real-world demographic data set revealed the ability of the GEOSOM to increase the spatial resolution of the clustering.

The GEO-SOM as presented by Bação et al. (2005a) is limited to two-dimensional geo-referenced data. In this study, the GEOSOM is extended to incorporate three-dimensional geo-referenced data, hence the name GEO3DSOM. The objective of the GEO3DSOM algorithm is to extent the exploratory data analysis capabilities of the standard SOM to incorporate the geographic location of the samples in three dimensions, based on the premise that samples located in each others vicinity are likely to be related to each other. A thorough discussion on the algorithms proposed is presented in the next section. Comparison between the standard SOM and the GEO3DSOM is carried out by applying both techniques to a theoretical data set and a hydrochemical data set from two phreatic, sandy aquifers in Central Belgium.

\section{Methods}

\subsection{Standard SOM}

Artificial Neural Networks (ANN) are computer algorithms, inspired by the functioning of the nervous system of the human brain, capable of learning from data and generalizing. This learning process can be described as supervised or unsupervised learning. In the supervised learning process, the ANN is shown several input-output patterns during training to enable the trained ANN to make generalizations based on the training data and to correctly produce output patterns based on new input (Jain et al., 1996). Neural networks are widely applied in hydrologic research (e.g. ASCE, 2000), especially in time-series prediction (e.g. Coppola et al., 2003; Alvisi et al., 2006)

The SOM-algorithm is based on unsupervised learning, which means that the desired output is not known a priori. The goal of the learning process is not to make predictions, but to classify data according to their similarity. In the early 1980s Kohonen proposed a neural network architecture in which the classification is done by plotting the data in ndimensions onto a, usually, two-dimensional grid of units in a topology-preserving manner (Kohonen, 1995). The former means that similar observations are plotted in each others neighborhood on the 2-D-grid. The network architecture and the learning algorithm are illustrated in Fig. 1.

The neural network consists of an input layer and a layer of neurons. The neurons or units are arranged on a rectangular or hexagonal grid and are fully interconnected. Each of the input vectors is also connected to each of the units. The learning algorithm applied to the network can be divided into six steps (Kohonen, 1995; Kaski, 1997):

1. An $m \times n$ matrix is created from the data set with $m$ rows of samples and $n$ columns of variables. The matrix thus consists of $m$ input vectors of length $n$. The classification of the input vectors is based on a similarity measurement, for instance Euclidean distance. In order to avoid bias in classification due to differences in measuring unit or range of the variables, a normalization is carried out. This can be done by setting mean equal to zero and variance equal to 1 or by rescaling the range of each variable in the $[0,1]$ interval.

2. Each unit is randomly assigned an initial weight or reference vector with a length equal to the length of the input vectors $(n)$.

3. An input vector is shown to the network; the Euclidean distances between the considered input vector $X$ and all of the reference vectors $M_{i}$ are calculated according to:

$$
\begin{aligned}
& X=\left(x_{1}, x_{2}, \ldots, x_{n}\right) \in \mathbb{R}^{n} \\
& M=\left(m_{1}, m_{2}, \ldots, m_{n}\right) \in \mathbb{R}^{n} \\
& \|X-M\|=\sqrt{\sum_{i=1}^{n}\left(x_{i}-m_{i}\right)^{2}}
\end{aligned}
$$

4. The best matching unit $M_{c}$, the unit with the greatest similarity with the considered input vector, is chosen according to:

$$
\left\|X-M_{c}\right\|=\min _{i}\left\{\left\|X-M_{i}\right\|\right\}
$$

This step is illustrated in Fig. 1b, where the Euclidean distance between the input vector $(0 ; 0.1 ; 0.02)$ and the reference vectors is calculated. The best matching unit is the upper left unit (distance $=0.102$ ). 
5. The weights of the best matching unit and the unit within its neighborhood $N(t)$ are adapted so that the new reference vectors lie henceforth closer to the input vector. The factor $\alpha(t)$ controls the rate of change of the reference vectors and is called the learning rate.

$$
M_{i}(t+1)= \begin{cases}M_{i}(t)+\alpha(t)\left[X(t)-M_{i}(t)\right] & \forall \in N(t) \\ M_{i}(t) & \forall \notin N(t)\end{cases}
$$

This is illustrated in Fig. 1c where the weights of the upper left unit and the units within the neighborhood $N(t)$ with radius $r$, indicated by the dashed line, are adapted. The rate of adaptation of the units is controlled by the neighborhood function $h$, which decreases from one at the winning unit to zero at units located farther away than radius $r$. The most common used functions are bell-shaped (Gaussian) or square (bubble).

6. Steps 3 until 5 are repeated until a predefined maximum number of iterations is reached. During these iterations both $\alpha$ and $N(t)$ decrease, forcing the network to converge.

After training, each of the input vectors is assigned to its best matching unit and the grids can be visualized. There are two types of grids commonly used to visualize and analyze the result of the SOM procedure: component planes and U-matrix (Vesanto et al., 1999). The U-matrix or distance matrix shows the Euclidean distance between neighboring units by means of a grey scale. Typically darker colors represent great distances and lighter shades represent small distances. In this visualization method clusters are represented by a light area with darker borders, meaning that the reference vectors in a cluster and the input vectors assigned to them are more similar to each other than to reference vectors outside the cluster. Additionally the labels of the input vectors can be plotted onto the U-matrix to identify the input vectors forming a cluster.

The component planes are the second visualization technique. In these maps the component values of the weight vectors are represented by a color code. Each of the component planes visualizes the distribution of one variable in the data set (Ultsch and Herrmann, 2005). By visually comparing those maps, variables with similar distributions can be detected and it helps in visually finding correlations between variables.

The Self-Organizing Map algorithm is closely related to $\mathrm{K}$-means clustering. A SOM with a number of units equal to the number of clusters in the data set and a neighborhood equal to zero will act as a traditional clustering technique (Kaski, 1997). A SOM may, however, be used in two very distinct ways: a large SOM, also known as emergent SOM, with many units, used for exploratory data analysis and cluster detection (Ultsch and Herrmann, 2005), and a small SOM for cluster centroid determination (Bação et al., 2005b). In
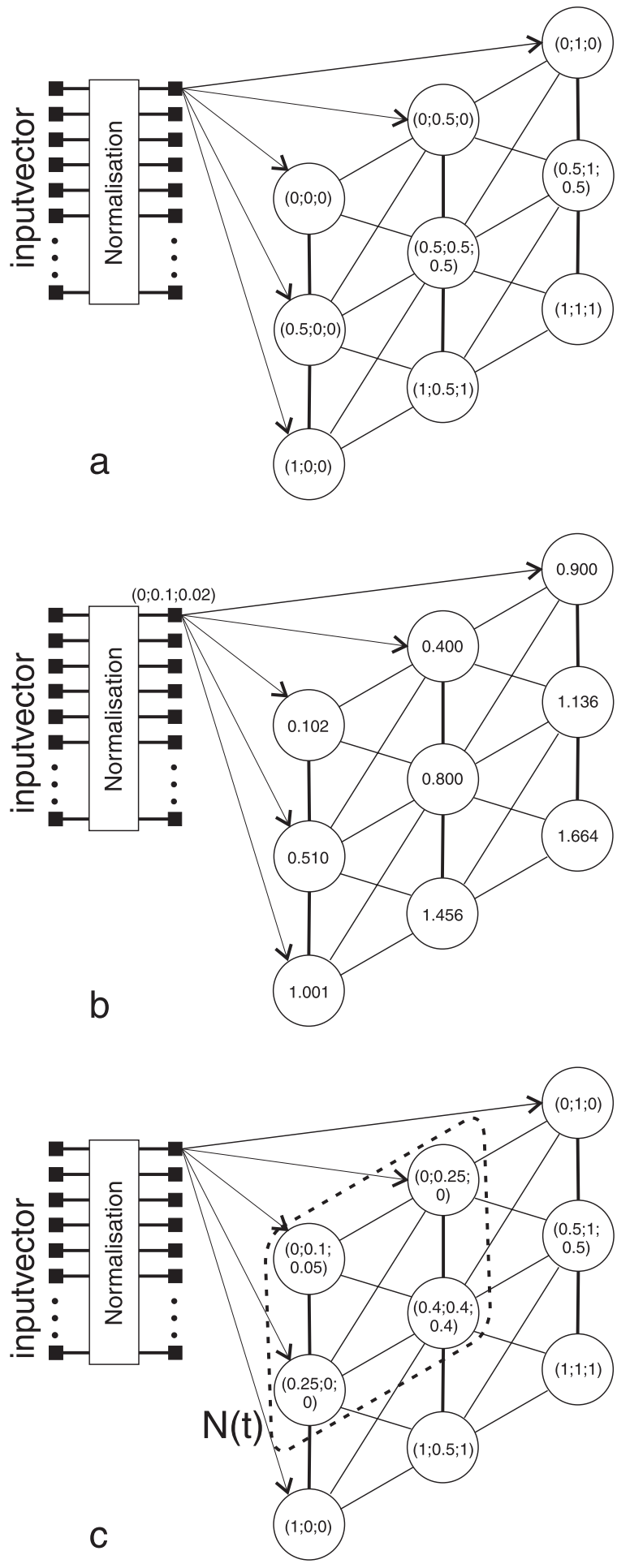

Fig. 1. SOM-algorithm

(a) Initialization reference vectors of the units

(b) Calculation of Euclidean distance between input vector and reference vectors of the units

(c) Assignment of input vector to its BMU and update of reference vectors of the units within neighborhood $\mathrm{N}(\mathrm{t})$. 


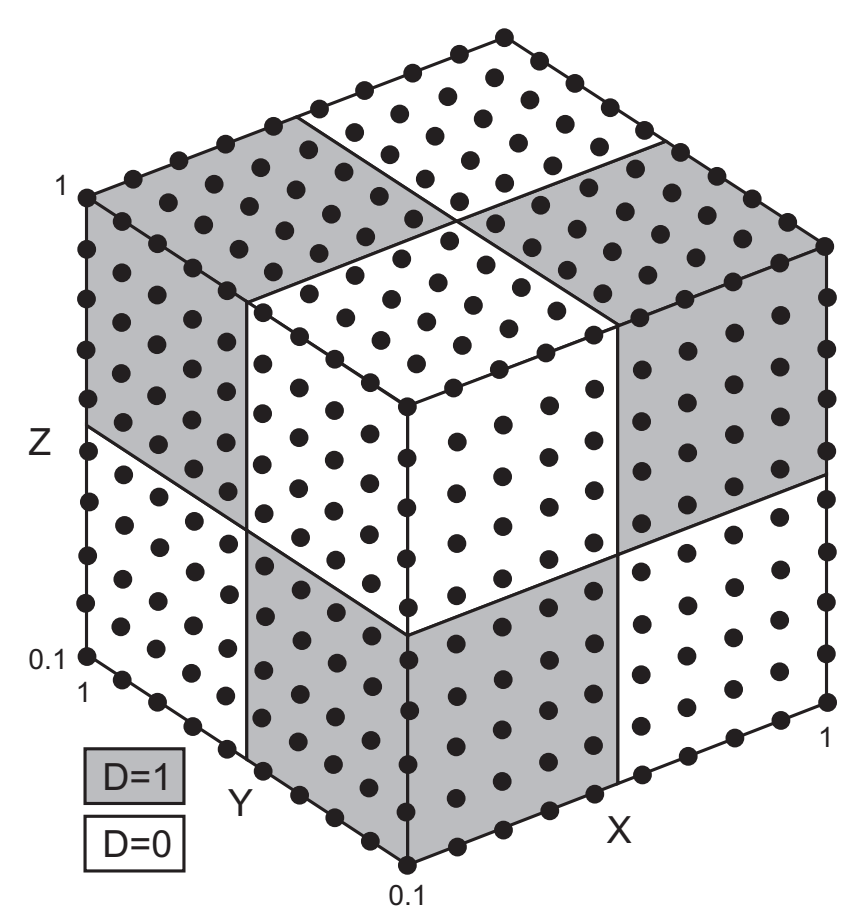

Fig. 2. Theoretical dataset.

this study, both SOM and GEO3DSOM are used for exploratory detection of clusters. When using K-means and fuzzy C-means-clustering, cluster centroids will always be detected based on the objective criterium of sum-of-squared distances. In emergent SOM's on the other hand, clearly separated groups of units may or may not be detected. Small SOM's used for centroid determination will act as a robust $\mathrm{K}$-means initialization in the first training iterations and due to the decrease of learning radius and neighborhood during training, the SOM will perform exactly as a K-means clustering in the final steps of the learning process (Bação et al., 2005b). Compared to K-means clustering and fuzzy c-means clustering, SOM has, in addition to the ability of SOM to directly visualize the results of the clusters in terms of the original variables, the advantage that the number of clusters does not need to be specified a priori. The advantage of K-means clustering and fuzzy c-means clustering on the other hand, is the ease of implementation since there are less parameters to be chosen. On the performance of clustering of SOM compared to other techniques a debate still exists in literature (overviews can be found in Bação et al., 2005b, and Mingoti and Lima, 2006). Provided the SOM is parameterized correctly, SOM will outperform K-means clustering since SOM is less sensitive to local optima compared to K-means Bação et al. (2005b).

\subsection{GEOSOM \& GEO3DSOM}

In the standard SOM-algorithm, geographic coordinates included in the data set are considered as any other variable.
The importance of the spatial variables during training of the map can be adjusted by assigning a weighting factor to these variables during the preprocessing stage. This procedure can be used to incorporate spatial information in the algorithm, although it has to be noted that samples located far from the center of the data set are ill represented in the SOM. In order to overcome this problem, Bação et al. (2005a) proposed the GEOSOM.

In the GEOSOM the spatial information of the data samples is explicitly included in the algorithm by altering the selection of the best-matching unit during the training into a two-step process. Firstly the unit is selected which lies geographically closest to the input vector. This means that the best-matching unit is searched based only on the geographic variables.

Secondly the unit with the smallest Euclidian distance, based on the complete input vector, within a predefined neighborhood of the geographically closest unit is chosen as best-matching unit. Subsequently the weight vector $M_{c}$ of the best-matching unit and the weight vectors $M_{i}$ within the neighborhood $N(t)$ are updated.

The size of the neighborhood to choose the best-matching unit from the units surrounding the geographically closest unit is determined by the variable $k$, the geographical tolerance. If $k$ equals zero, the best-matching unit is the geographically closest unit. Setting $k$ greater than zero, results in the search of the best-matching unit among the units within a radius $k$ in output space of the geographically closest unit. If $k$ approaches the size of the map, the result is equal to that of the standard SOM-algorithm.

The GEOSOM is only capable of including two geographic coordinates in the selection of the geographically closest unit, namely the $\mathrm{X}$ and $\mathrm{Y}$ coordinate. The GEO3DSOM is an extended version of the GEOSOM, capable of incorporating the third dimension, $\mathrm{Z}$, in the selection of the geographically closest unit.

In order to give each geographic coordinate equal weight in the training process, each of the coordinate variables is rescaled so that their ranges are comparable, e.g. between $[0,1]$.

The GEO3DSOM-algorithm is implemented in Matlab ${ }^{\circledR}$ compatible with the SOM-toolbox (Vesanto et al., 1999).

\section{Results}

In the following section the standard SOM and the GEO3DSOM are applied to a theoretical data set and a real world hydrochemical data set.

\subsection{Theoretical data set}

The dataset consists of 1000 points of a cube, regularly spaced with an interval of 0.1 between $[0.1,1]$. A variable $\mathrm{D}$ was added to this dataset with a value of 0 or 1 . The 
U-matrix

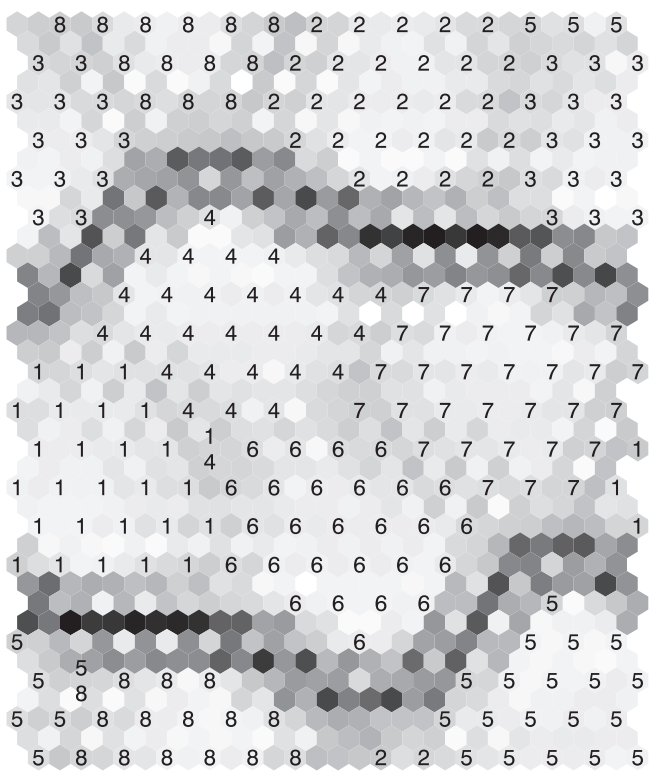

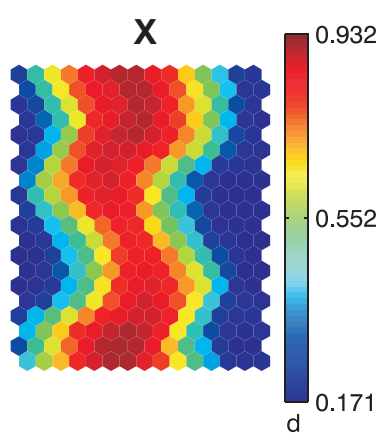
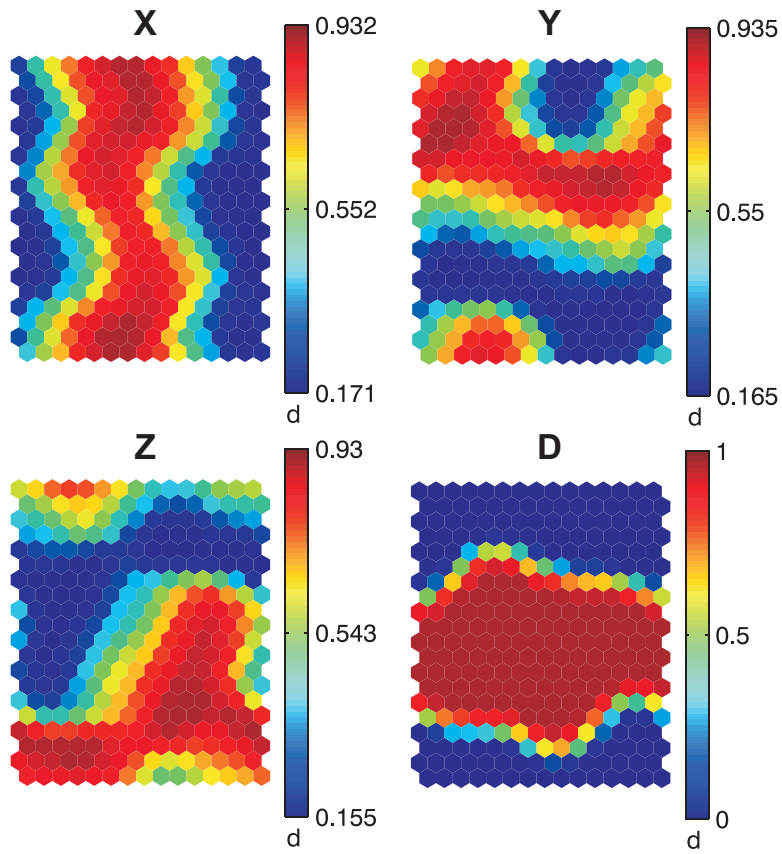

Fig. 3. U-matrix (left) and component planes (right) of the standard SOM-analysis of the theoretical data set.
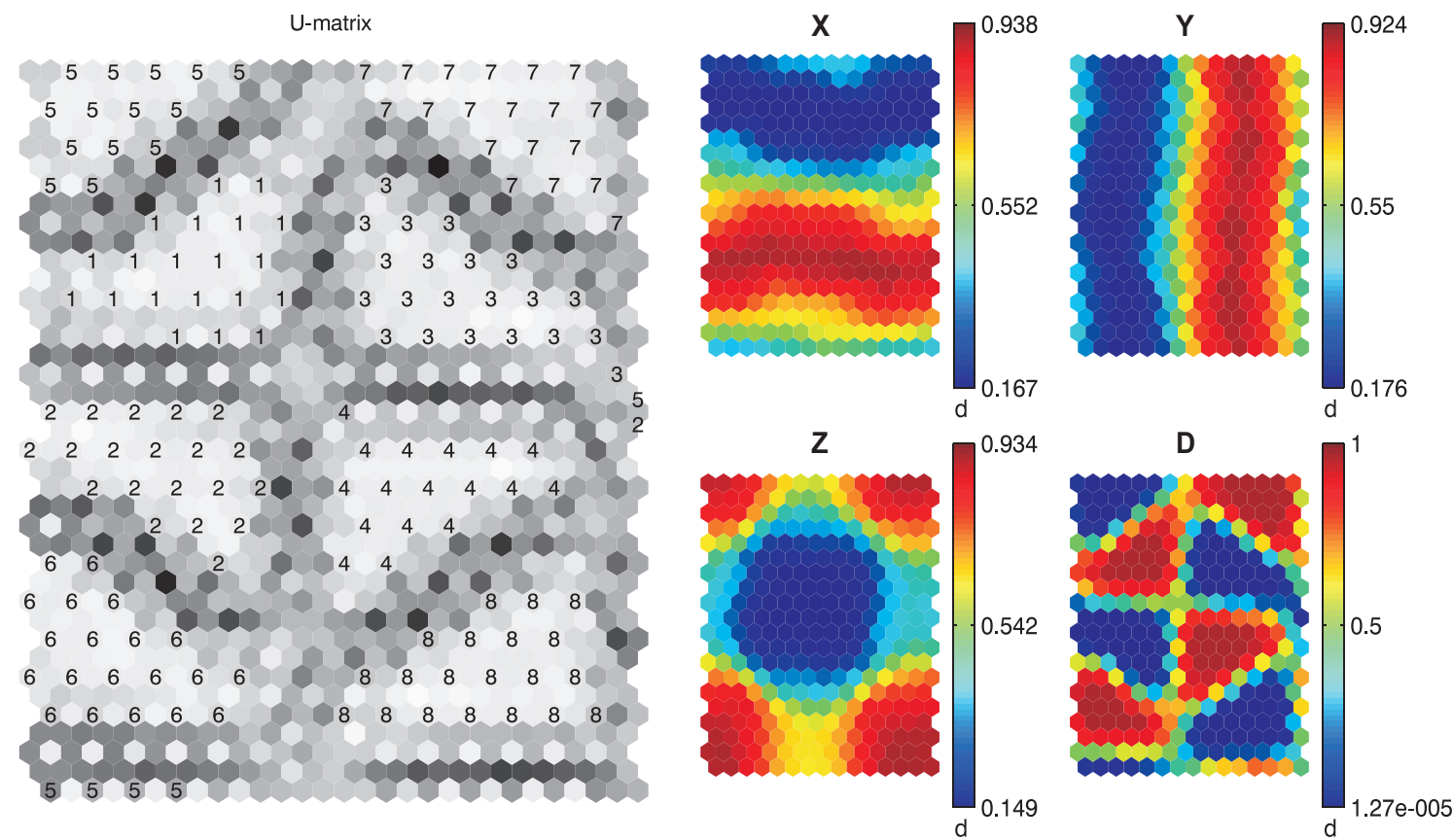

Fig. 4. U-matrix (left) and component planes (right) of the standard GEO3DSOM-analysis of the theoretical data set.

distribution of variable $\mathrm{D}$ is shown in Fig. 2. This distribution results in 8 pre-defined groups (Table 1).

This data set is analyzed with both the standard SOM and the GEO3DSOM. The parameters used in the analysis are summarized in Table 2.
In both SOM-analysis the grid consists of 20 by 15 units, hexagonally ordered on a toroid shape. The use of rectangular array with a large number of units allows a good representation of the topology of the data set, while the hexagonal ordering provides more neighbors to each unit and border effects are avoided by using the continuous, finite shape of a toroid (Ultsch and Herrmann, 2005). 
Table 1. Pre-defined groups in the theoretical data set.

\begin{tabular}{ccccc}
\hline $\mathrm{X}$ & $\mathrm{Y}$ & $\mathrm{Z}$ & $\mathrm{D}$ & Pre-defined groups \\
\hline $0.1-0.55$ & $0.1-0.55$ & $0.1-0.55$ & 1 & 1 \\
$0.55-1$ & $0.1-0.55$ & $0.1-0.55$ & 0 & 2 \\
$0.1-0.55$ & $0.55-1$ & $0.1-0.55$ & 0 & 3 \\
$0.55-1$ & $0.55-1$ & $0.1-0.55$ & 1 & 4 \\
$0.1-0.55$ & $0.1-0.55$ & $0.55-1$ & 0 & 5 \\
$0.55-1$ & $0.1-0.55$ & $0.55-1$ & 1 & 6 \\
$0.1-0.55$ & $0.55-1$ & $0.55-1$ & 1 & 7 \\
$0.55-1$ & $0.55-1$ & $0.55-1$ & 0 & 8 \\
\hline
\end{tabular}

Table 2. Parameters of SOM and GEO3DSOM-analysis.

\begin{tabular}{|c|c|c|}
\hline Parameter & Standard SOM & GEO3DSOM \\
\hline $\begin{array}{l}\text { size } \\
\text { grid } \\
\text { type } \\
h \\
\text { training mode }\end{array}$ & $\begin{array}{l}20 \times 15 \\
\text { hexagonal } \\
\text { toroid } \\
\text { bubble } \\
\text { sequential }\end{array}$ & $\begin{array}{l}20 \times 15 \\
\text { hexagonal } \\
\text { toroid } \\
\text { bubble } \\
\text { sequential }\end{array}$ \\
\hline $\begin{array}{l}\text { rough training } \\
\text { epochs } \\
r_{\text {initial }} \\
\alpha_{\text {initial }}\end{array}$ & $\begin{array}{l}50 \\
15 \\
0.7\end{array}$ & $\begin{array}{l}50 \\
15 \\
0.7\end{array}$ \\
\hline $\begin{array}{l}\text { fine training } \\
\text { epochs } \\
r_{\text {initial }} \\
\alpha_{\text {initial }}\end{array}$ & $\begin{array}{l}50 \\
4 \\
0.1\end{array}$ & $\begin{array}{l}50 \\
4 \\
0.1\end{array}$ \\
\hline$k$ & - & 2 \\
\hline
\end{tabular}

Figures 3 and 4 show respectively the results of the standard SOM-analysis and the GEO3DSOM-analysis. The U-matrices show the Euclidean distances between the reference vectors of the units by means of a gray scale (black: large distance, white: small distance). The units of the Umatrices are labeled with the cluster number of the sample assigned to the unit, according to Table 1.

Visual inspection of the U-matrices shows that both SOManalysis are able to extract the clusters from the data. In the U-matrix of standard SOM-analysis a clear separation between groups is only visible between the clusters with $D=1$ and the clusters with $D=0$. Although the samples are grouped according to the pre-defined groups, no distinct borders between these groups are present. The U-matrix of the GEO3DSOM-analysis on the other hand, clearly shows that each cluster is separated from another one by a zone of high Euclidian distance between reference vectors.

The accompanying component planes can be used to explore the differences between the clusters. From both Figs. 3 and 4 it can be seen that the area with the samples of cluster 4
Table 3. Quality measures for the theoretical data set.

\begin{tabular}{lll}
\hline Quality measure & standard SOM & GEO3DSOM \\
\hline qe & 0.115 & 0.145 \\
te & 0.128 & 0.070 \\
ge & 0.100 & 0.097 \\
\hline
\end{tabular}

assigned to it, is characterized by $X>0.55, Y>0.55, Z<0.55$ and $D=1$.

In order to asses the quality of the SOM-analysis in representing the data set, three quality measures can be computed; the quantization error ( $q e)$, the topographic error ( $t e$ ) and the geographic error $(g e)$. The quantization error measures the resolution of the SOM and is calculated as the average Euclidian distance between an input vector and the reference vector of its best matching unit (Kohonen, 1995). The topographic error quantifies the preservation of the topology of the data by calculating the proportion of all data vectors for which first and second best matching unit are not adjacent units (Kohonen, 1995). Finally, the geographic error is a measure for the ability of the SOM to represent the geographic distribution of the data samples. It is calculated as the geographic distance, the Euclidean distance calculated based on the $X, Y$ and $Z$ coordinates, between an input vector and its best matching unit. Table 3 summarizes the quality measures for the standard SOM-analysis and the GEO3DSOManalysis.

The quantization error for the standard SOM is lower than for the GEO3DSOM, meaning that the representation of samples is better in the standard SOM. The GEO3DSOM, on the other hand, scores better in terms of topographic and geographic error. The representation of data by the GEO3DSOM is thus better capable of capturing the topology of the data and the geographic information included in the data.

\subsection{Hydrochemical data set}

The hydrochemical data set is obtained from a monitoring network of the Flemish Government in two regional, sandy, phreatic aquifers, made available through Databank Ondergrond Vlaanderen (DOV, 2006). The data set consists of 47 observation wells, each equipped with three well screens at different depths, resulting in a data set of 131 samples. Facilities in the monitoring well are designed to allow independent sampling of discrete depth intervals without mixing of groundwater of different depths.

The first aquifer, the Diest sands aquifer is of Late Miocene age and consists of coarse, glauconiferous sands and sandstones (Laga et al., 2001). The Brussels sands aquifer is of Middle Eocene age and is a heterogeneous formation consisting of an alteration of highly and poorly calcareous sands, which are locally silicified (Laga et al., 2001). 


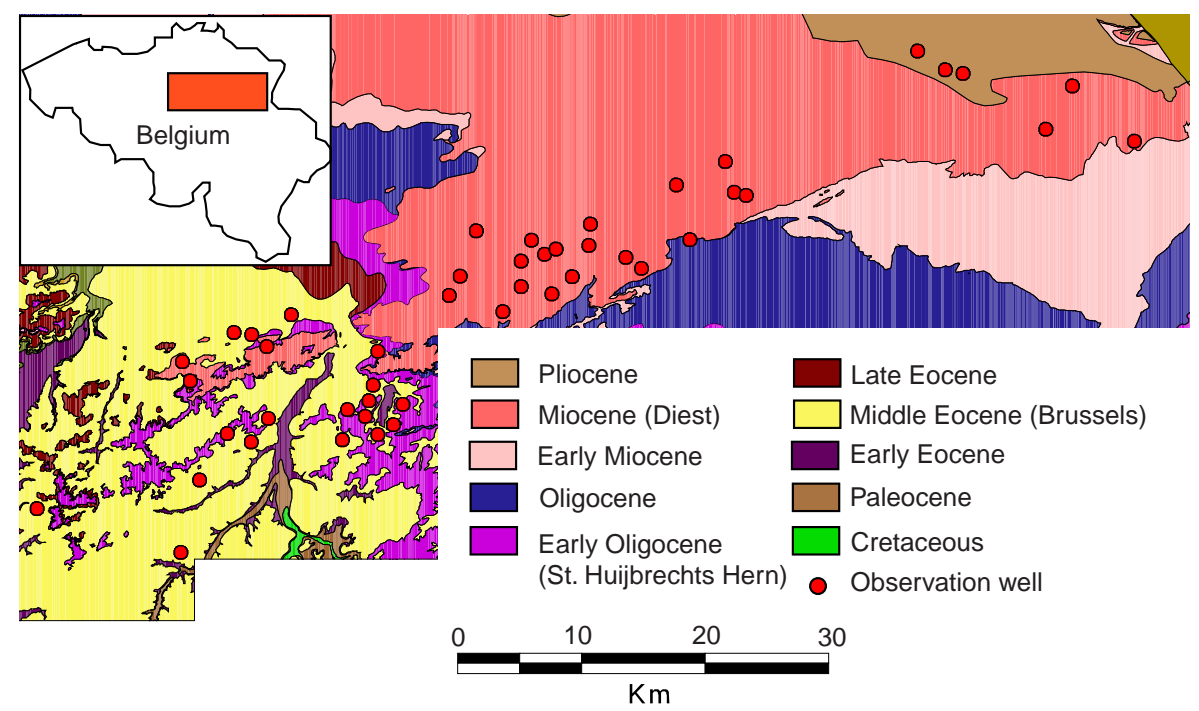

Fig. 5. Study area (after DOV, 2006).

Locally the Brussels sands are overlain by the younger sandy formations of Lede (Middle Eocene) and St. Huibrechts Hern (Early Oligocene). Both aquifers are covered with Quaternary eolian deposits consisting mainly of sands in the north and loam in the south.

Figure 5 shows the geological map of the study area and location of piezometers used in this study.

A sampling campaign was carried out in the spring of 2005 and from the 20 measured variables, a subset of 12 variables are considered in this analysis. Geographic coordinates, X, Y and the $\mathrm{Z}$ position above sealevel of the filter of each sample are included in the data set.

Histograms of the variables (Fig. 6) show that most of the variables are not normally distributed, but rather have a bimodal $\left(\mathrm{Ca}^{2+}, \mathrm{pH}\right.$ and $\left.\mathrm{HCO}_{3}^{-}\right)$, skewed (e.g. $\mathrm{K}^{+}, \mathrm{Mg}^{2+}$, $\mathrm{O}_{2}$ and $\left.\mathrm{NO}_{3}^{-}\right)$or even a lognormal distribution $\left(\mathrm{Fe}^{2+/ 3+}\right.$ and $\mathrm{Mn}^{2+}$ ). In order to avoid bias in the normalization or to make assumptions regarding the distribution of the variables, all parameters, including $\mathrm{X}, \mathrm{Y}, \mathrm{Z}$ are rescaled to a $[0,1]$ interval, according to:

$x_{\text {new }}=\frac{x_{\text {old }}-\min (X)}{\max (X)-\min (X)}$

A standard SOM analysis and a GEO3DSOM-analysis are carried out on the normalized data set. The parameters used in both analysis are summarized in Table 4 .

The number of units, the number of iterations and the kvalue are determined based on a sensitivity analysis. The results of this sensitivity analysis are rendered in Fig. 7. In the sensitivity analysis only the value of the variable under study is changed, while the other variables are set to the values given in Table 4. The number of grid nodes to be used in a SOM-analysis can be considered as a trade-off between representation accuracy and generalization accuracy. A small
Table 4. Parameters of SOM and GEO3DSOM-analysis.

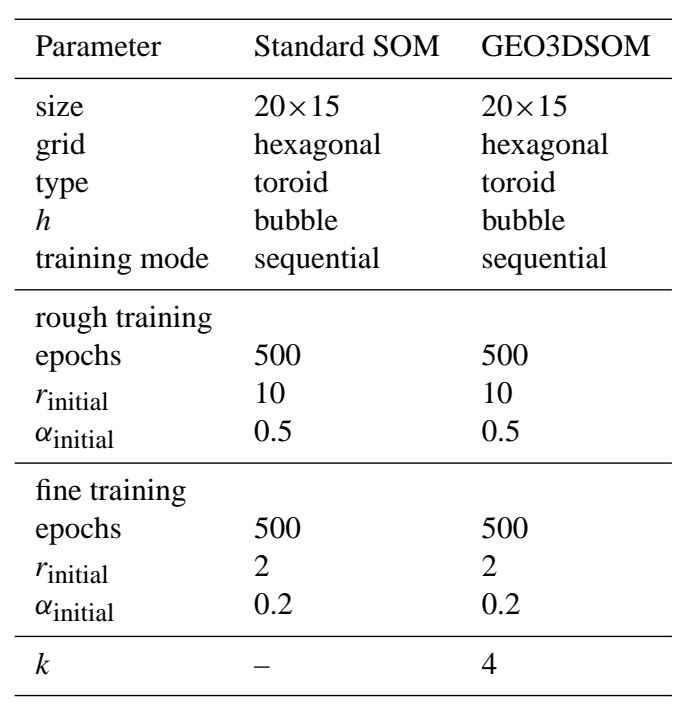

number of grid nodes will result in a high quantization error and well-defined clusters, while a large number of nodes result in a low quantization error and, in the most extreme case, a cluster for each data sample. Figure 7 a shows the evolution of quantization, topologic and geographic error for maps with different numbers of units. The grid configuration is rectangular for each of the maps, based on a 3 by 4 configuration. Each of the resulting maps were visually evaluated for their clustering ability. The quantization error rapidly decreases with increasing number of grid nodes, while the topologic and geographic error converge to a stable value (Fig. 7a). The best compromise between clustering ability 

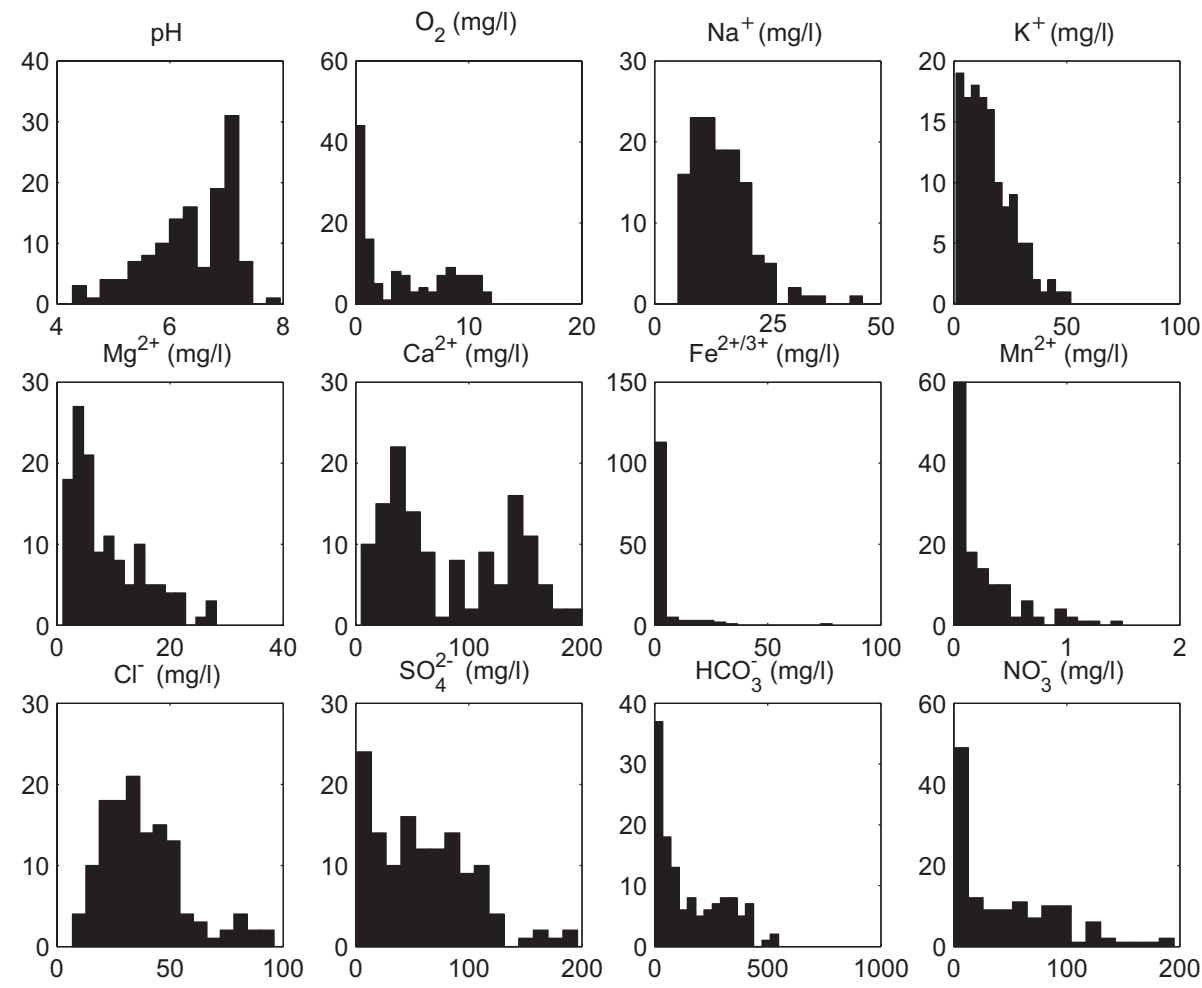

Fig. 6. Histograms of the hydrochemical data set.

Table 5. Quality measures for the hydrochemical data set.

\begin{tabular}{lll}
\hline Quality measure & standard SOM & GEO3DSOM \\
\hline qe & 0.127 & 0.139 \\
te & 0.076 & 0.160 \\
ge & 0.028 & 0.024 \\
\hline
\end{tabular}

and quantization error was found for the 20 by 15 nodes configuration (300 nodes). Figure $7 \mathrm{~b}$ shows the influence of the number of iterations on the quantization, topologic and geographic error. The number of iterations shown are the total number of iterations after rough and fine training. After 1000 iterations (500 rough training and 500 fine training) the topologic and geographic error are stabilized, while the quantization error only decreases slightly. The sensitivity analysis with regards to the k-value (Fig. 7c) reveals that with increasing k-value, the geographic error increases rapidly and the quantization error decreases. Once again a compromise has to be found between the weight given to the geographic coordinates and the overall quantization. After visual examination of the ability of the different maps to represent the data, a k-value of 4 produced the best results both with respect to low quantization and geographic error and with respect to visualizing and grouping of the data. The choice of 4 for the geographic tolerance $k$ implies that the search of the BMU

is restricted to the units lying within a radius of 4 units surrounding the geographically closest unit.

The results of both analysis are depicted in Fig. 8 (standard SOM) and Fig. 9 (GEO3DSOM). The visualized results are (a) component planes, (b) U-matrix labeled with geology (B: Brussel sands, S: St. Huybrechts Hern sands, D: Diest sands, Q: Quaternary deposits), (c) false coloring of the SOM based on the U-matrix and finally (d) spatial distribution well screens, colored using the false coloring. The spatial distribution of the groups is organized per well screen, with screen 1 being the shallowest screen and screen 3 the deepest. The false coloring of the units of the self-organizing map is carried out in such a way that units with similar weight vectors have similar colors, using a naive contraction model based on the U-matrix according to the algorithm proposed by Himberg (2000). The resulting color coding is then applied to the representation of the well screens. In this way a fuzzy ordering of the well screens is carried out, linking them through the false colored SOM with the component planes, without having to manually delineate groups.

Table 5 renders the quality measures for both analysis. For the standard SOM the quantization error is slightly lower than the $q e$ of the GEO3DSOM. The topologic error on the other hand is significantly lower for the standard SOM than for the GEO3DSOM. The performance of the standard SOM in capturing and representing the structure of the data set is higher than the performance of the GEO3DSOM. This is 

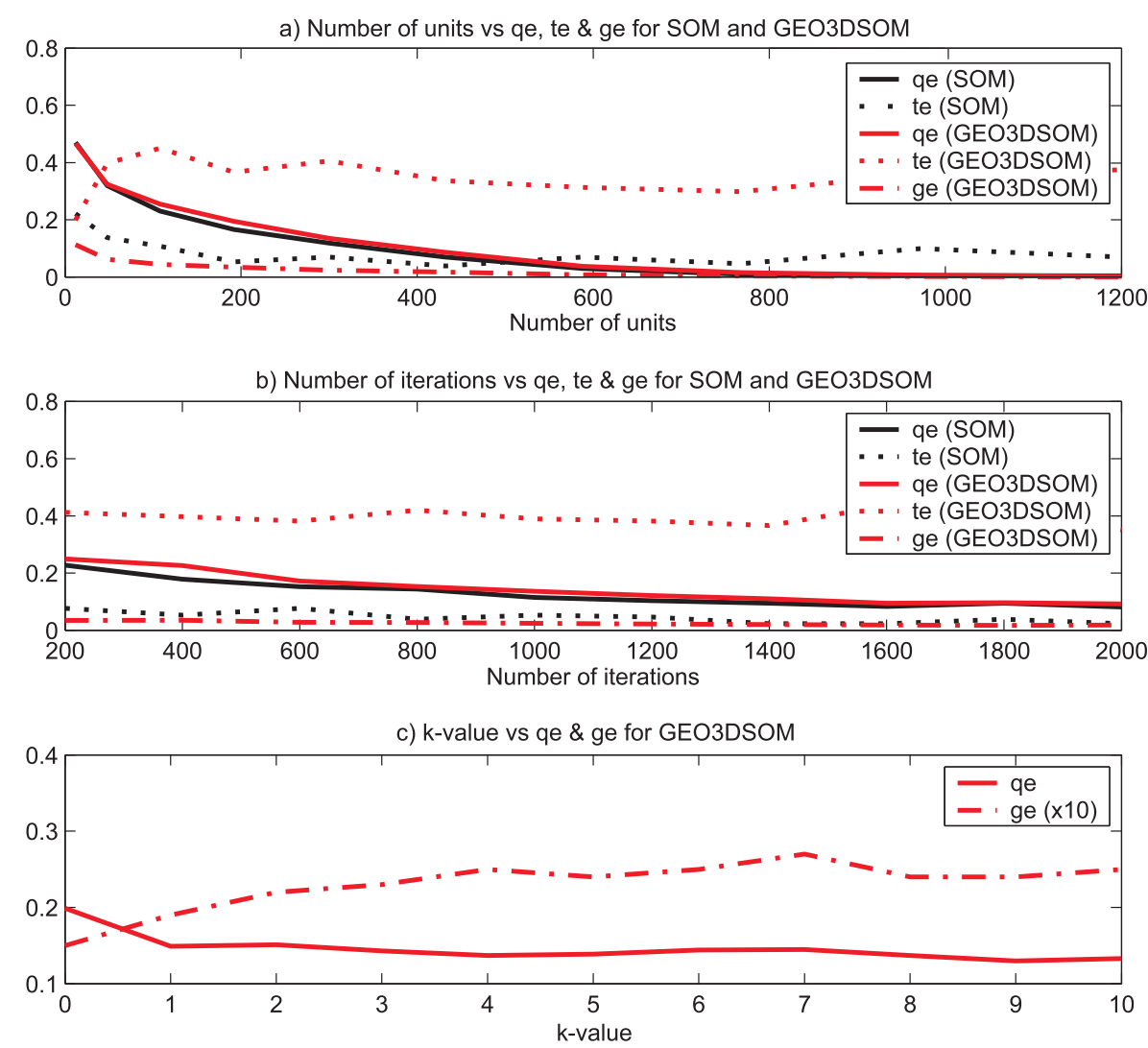

Fig. 7. Results of the sensitivity analysis with respect to (a) the number of units, (b) the number of iterations and (c) the value of the geographical tolerance $k$ for SOM and GEO3DSOM.

noticeable on the component planes (Fig. 8a and Fig. 9a), where distributions of the variables on the component planes of the standard SOM are rather smooth compared to those of the GEO3DSOM. This is mainly due to the better topological representation of the standard SOM.

The geographic error of the GEO3DSOM, however, is $15 \%$ smaller than in the standard SOM, implying that the geographic representation of the GEO3DSOM resembles the data set more closely.

On the U-matrices (Figs. $8 b$ and $9 b$ ), it is also noticeable that the U-matrix of the GEO3DSOM divides the SOM in a large number of well separated groups, while the number of groups in the standard SOM is smaller and the borders between groups are less distinct. The more distinct borders between groups in the GEO3DSOM U-matrix results in a higher resolution for the false coloring compared to the standard SOM coloring (Figs. 8c and 9c).

Both SOM-variants succeed in distinguishing between samples originating from the Diest and the Brussels aquifers, as can be deduced from the geology-labeled U-matrices (Figs. $8 \mathrm{~b}$ and $9 \mathrm{~b}$ ). The component planes reveal that $\mathrm{pH}$ and concentrations of calcium and bicarbonate are relatively high in the Brussels aquifer. This difference is due to the presence of calcite in the Brussels sands, while calcite is almost absent in the Diest sands (Laga et al., 2001; Lagrou et al., 2004). In the false coloring of the GEO3DSOM this geology related subdivision is clearly visible through a distinct difference in coloring. In the coloring of the SOM however the distinction between Diest and Brussels samples is, if present, only noticeable through subtle color differences.

The Quaternary samples can also be differentiated from the rest, albeit less clear, since the Quaternary samples are characterized by an overall very low mineralization, with the exception of nitrate and chloride which can be locally very high. Since these well screens are rather shallow and the Quaternary deposits consist of sands and gravels, the composition of groundwater is very close to the composition of rain water, hence the low $\mathrm{pH}$, and very susceptible to anthropogenic influences like chlorine and nitrate contamination. In both SOM variants the region with Quaternary samples is apparent from the false coloring. A smaller variation in groundwater chemistry can be detected in the Brussels aquifer, where the most northern and the north eastern samples are characterized by lower $\mathrm{Mg}^{2+}$ concentrations. In the coloring of the SOMs this is noticeable through subtle difference in shades of green.

Further subdivision of the SOM's is possible based on the concentrations of oxygen, nitrate, iron and manganese. In 


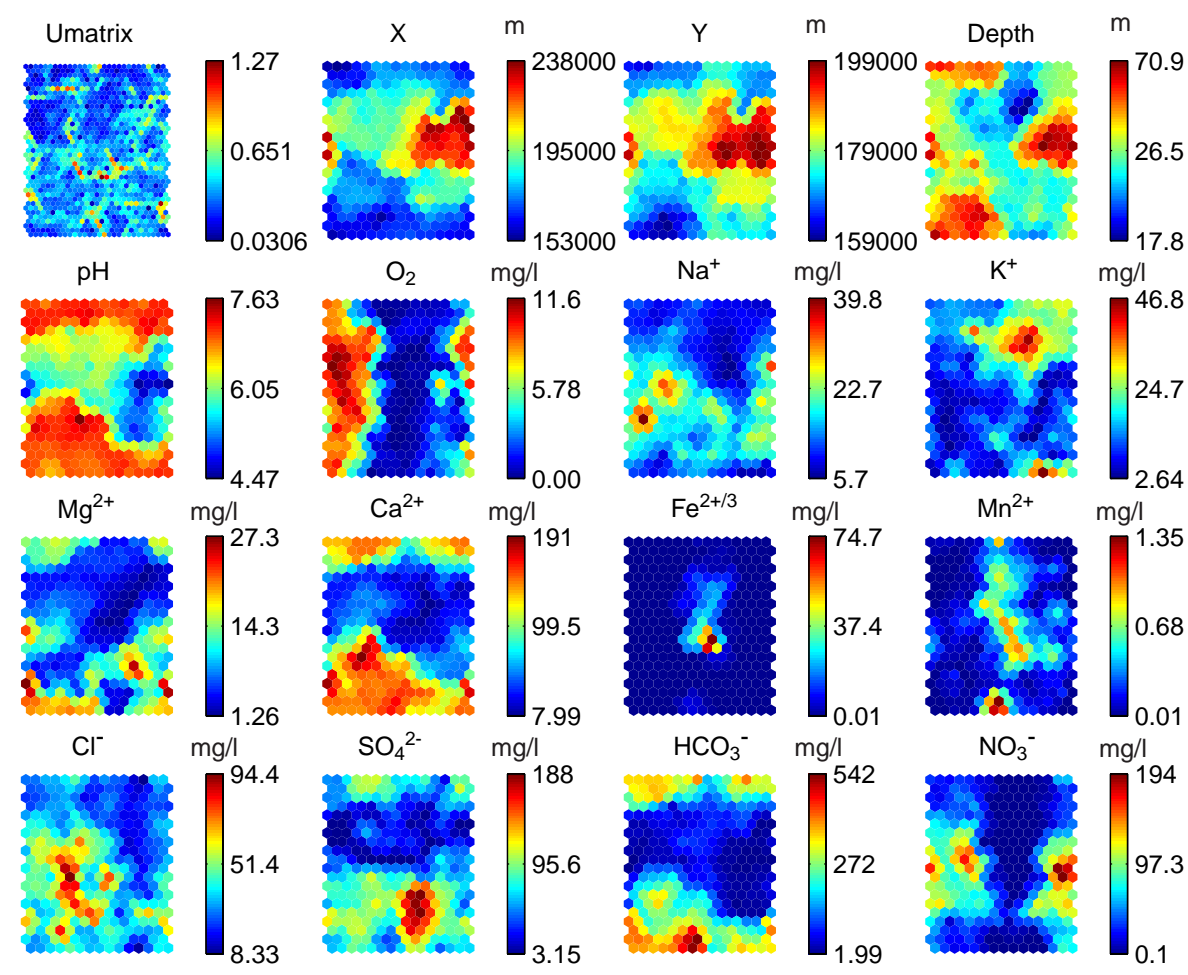

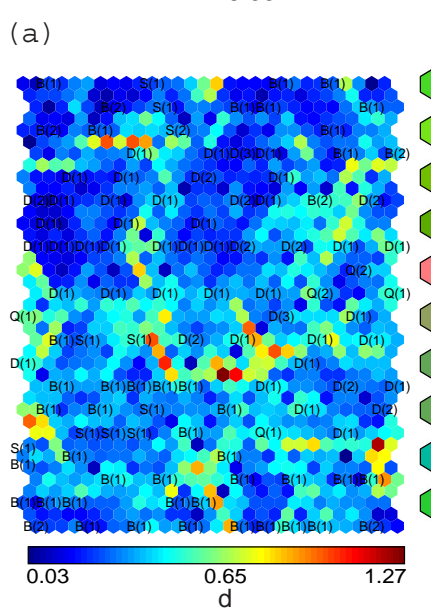

(b)

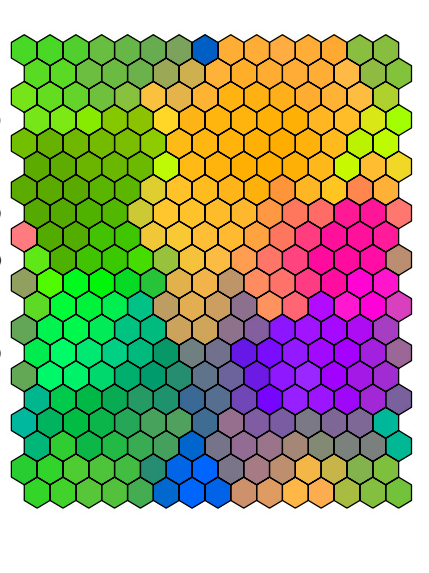

(c)

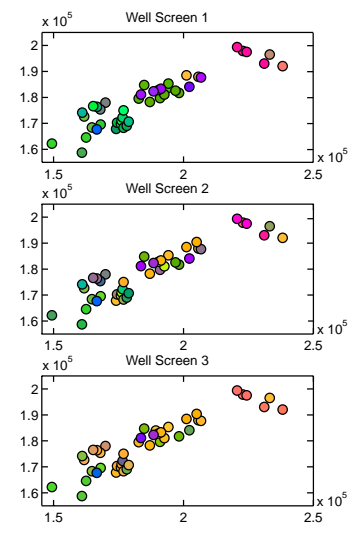

(d)

Fig. 8. Results of the SOM-analysis of the hydrochemical data set.

(a) U-matrix and component planes

(b) labeled U-matrix

(c) false coloring of U-matrix

(d) spatial distribution of well screens, using false coloring.

both aquifers there are zones with low oxygen concentrations and elevated iron and manganese. These groups consist of the deeper samples (Figs. 8d and 9d) and nitrate concentrations are on average lower in these groups. Due to the ubiquitous presence of iron and manganese bearing minerals like glauconite and iron-oxides in the Diest aquifer (Lagrou et al., 2004), the iron concentrations are rather high in the
Diest aquifer when oxygen concentrations are low. This subdivision of the data is determining the color coding of the standard SOM, while in the GEO3DSOM this feature is subordinate to the coloring based on $\mathrm{pH}, \mathrm{Ca}^{2+}$ and $\mathrm{HCO}_{3}^{-}$.

In the Brussels sands aquifer there are three well screens, located in the same well, with anomalous high manganese concentrations and low oxygen and nitrate concentrations. 


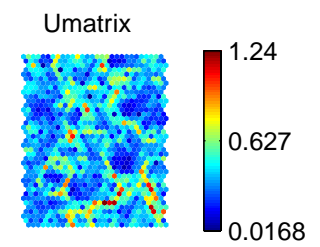

$\mathrm{pH}$

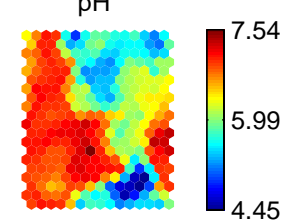

$\begin{array}{ll}\mathrm{Mg}^{2+} & \mathrm{mg} / \mathrm{l} \\ 27.7\end{array}$

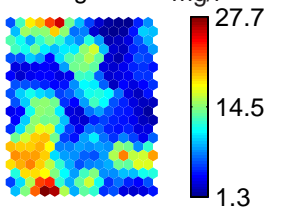

$\mathrm{Cl}^{-}$

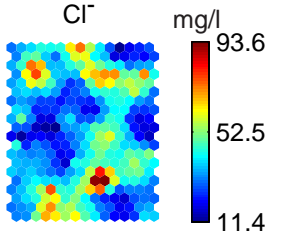

(a)

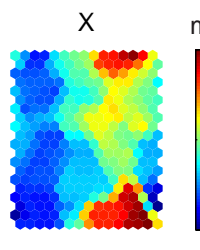

$\mathrm{O}_{2}$

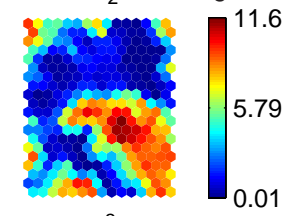

$\mathrm{Ca}^{2+}$

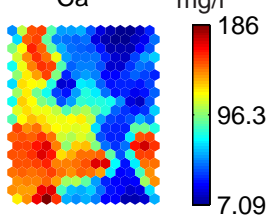

$\mathrm{SO}_{4}^{2-}$

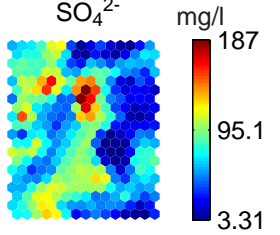

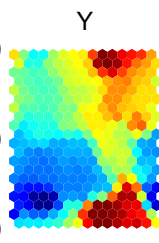

$\mathrm{Na}^{+}$

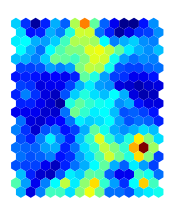

$\mathrm{Fe}^{2+/ 3+}$

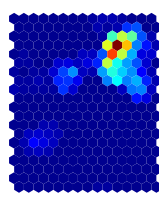

$\mathrm{HCO}_{3}^{-}$

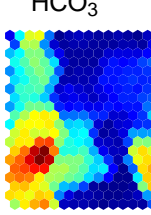

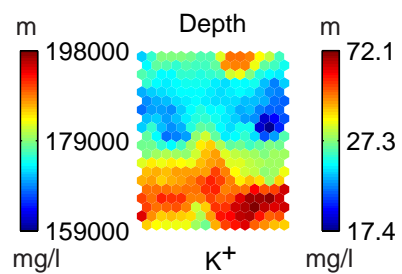

38.9

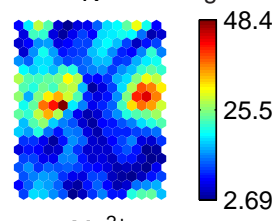

$\mathrm{Mn}^{2+} \mathrm{mg} / \mathrm{l}$

$\mathrm{mg} / \mathrm{l}$

66.5
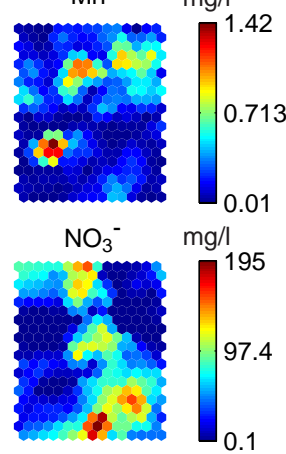

33.3

001

$\mathrm{mg} / \mathrm{l} / \mathrm{s}$
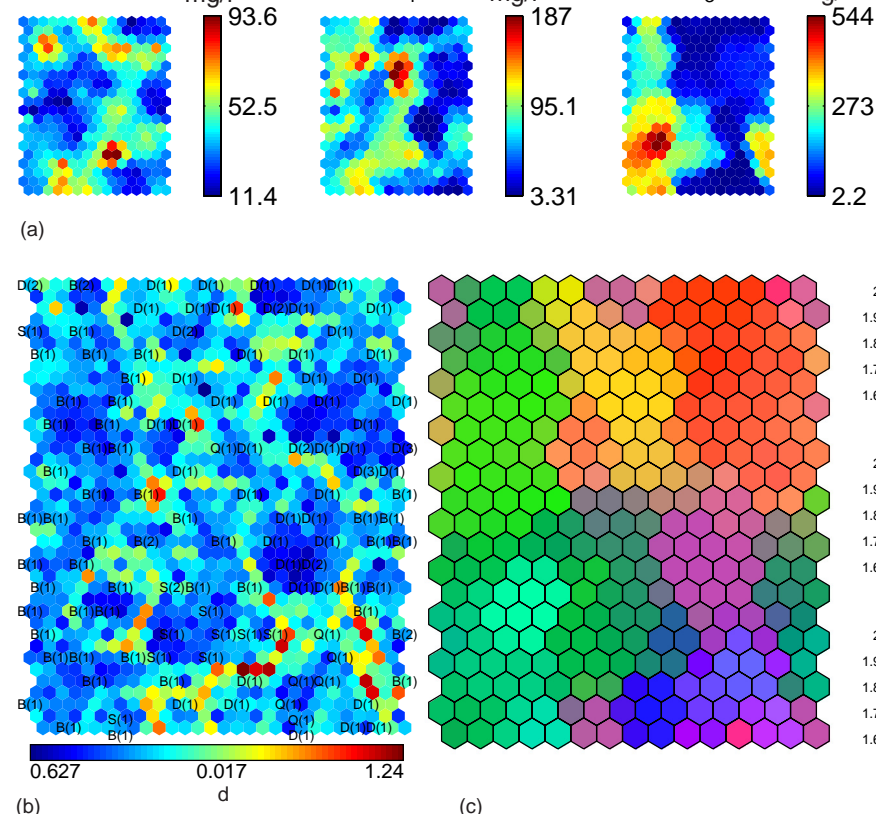

(c)

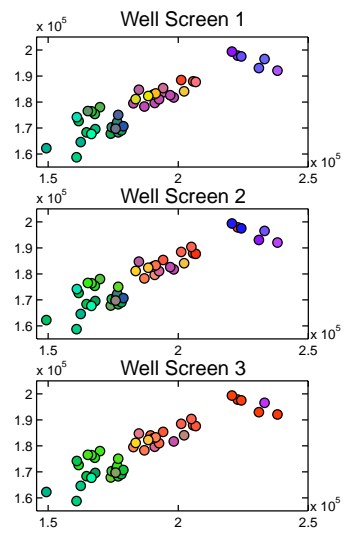

(d)

Fig. 9. Results of the GEO3DSOM-analysis of the hydrochemical data set.

(a) U-matrix and component planes

(b) labeled U-matrix

(c) false coloring of U-matrix

(d) spatial distribution of well screens, using false coloring.

Both SOM-variants succeed in isolating and identifying these outlying values.

\section{Conclusions}

The self-organizing map algorithm has proved to be a very valuable tool in the visualization and interpretation of large, multivariate data sets.
To incorporate spatial information in a self-organizing map analysis, GEO3DSOM is developed and its performance in clustering of both an artificial and a real life data set is compared to the standard SOM.

The performance of the standard SOM in correctly representing the structure of the data set and in minimizing the error between the input vectors and its best matching unit is higher than the performance of the GEO3DSOM on these criteria. The standard SOM is therefore very suitable for an 
exploratory data analysis in order to capture relationships between variables and the structure of data.

The GEO3DSOM on the other hand outperforms the standard SOM in providing a grouping of the data in a spatially coherent way. Analysis of both the artificial and the real life data sets showed that the GEO3DSOM is capable of a more detailed grouping of both regularly and irregularly distributed spatial data, compared to the standard SOM with geographical coordinates included in the data set. As is to be expected, the information about the data set at hand obtained through the component planes and the U-matrices by both versions of the SOM is very similar. The pseudocoloring applied to both variants of the SOM-algorithm however shows some clear differences between both techniques. The explicit incorporation of the geographic coordinates in the GEO3DSOM algorithm results in greater differences between groups in the U-matrix. This results in an increased resolution in the pseudo-coloring of the units. In the GEO3DSOM both the geology related subdivision and the vertical subdivision is apparent from the coloring, while in the standard SOM the coloring is dominated only by the vertical subdivision based on oxygen, iron and manganese. Within the samples having elevated oxygen and nitrate concentrations, a subtle differentiation between Brussels and Diest samples can be seen, while this differentiation is completely absent in the group of samples with low oxygen and nitrate concentrations. Both coloring schemes do however identify the presence of outliers in the Brussels sands aquifer. In conclusion it can be stated that both techniques succeed very well in providing more insight in the quality data set, highlighting the main differences and pointing out anomalous wells. Incorporation the spatial correlation through including the geographic coordinates in the BMU-selection procedure of GEO3DSOM, however, provides the advantage of an increased resolution, while still maintaining a generalization of the data set.

Acknowledgements. The authors wish to thank AMINAL for providing data through their website (DOV, 2006).

Edited by: D. Solomatine

\section{References}

Alvisi, S., Mascellani, G., Franchini, M., and Bardossy, A.: Water level forecasting through fuzzy logic and artificial neural network approaches, Hydrol. Earth Syst. Sci. 10, 1-17, 2006.

ASCE Task Committee on Application of Artificial Neural Networks in Hydrology: Artificial neural networks in hydrology. II: Hydrologic applications, J. Hydrol. Eng., 5(2), 124-137, 2000.

Bação, F., Lobo, V., and Painho, M.: The self-organizing map, the Geo-SOM, and relevant variants for geosciences, Computers and Geosciences, 31(2), 155-163, 2005a.

Bação, F., Lobo, V., and Painho, M.: Self-organizing maps as substitute for K-means clustering, in: International conference on computational science, edited by: Sunderarm, V. S., van Albada, G., Sloot, P., and Dongarra, J. J., International conference on computational science 2005, Lecture Notes in Computer Science, Springer-Verlag Berlin, Berlin, 3516, 476-483, 2005 b.

Chang, H. C., Kopaska-Merkel, D. C., and Chen H. C.: Identification of lithofacies using Kohonen self-organizing maps, Computers and Geosciences, 28(2), 223-229, 2002.

Coppola, E., Szidarovsky, F., Poulton, M., and Charles, E.: Artificial neural network approach for predicting transient water levels in a multilayered groundwater system under variable state, pumping and climate conditions, J. Hydrol. Eng., 8(6), 348-360. 2003.

Davis, J. C.: Statistics and data analysis in geology, John Wiley \& Sons, Inc, New York, 1986.

Databank Ondergrond Vlaanderen: http://dov.vlaanderen.be, 2006.

Güler, C., Thyne, G. D., and McCray, J. E.: Evaluation of graphical and multivariate statistical methods for classification of water chemistry data, Hydrogeology J., 10(4), 455-474, 2002.

Himberg, J.: A SOM Based Cluster Visualization and Its Application for False Coloring, Proceedings of International Joint Conference on Neural Networks (IJCNN2000), 3, 587-592, 2000.

Hong, Y. S. and Rosen, M. R.: Intelligent characterisation and diagnosis of the groundwater quality in an urban fractured-rock aquifer using an artificial neural network, Urban Water, 3(3), 193-204, 2001.

Jain, A. K., Mao, J., and Mohiuddin, K.: Artificial Neural Networks: a tutorial, IEEE Computer, 26(3), 31-44, 1996.

Kaski, S.: Data exploration using Self-Organizing Maps, Acta Polytechnica Scandinavica: Mathematics, computing and management in engineering, Series No 82, 57, 1997.

Kohonen, T.: Self-organizing maps. Springer, Berlin, 1995.

Koua, E. L., Maceachren, A., and Kraak, M.-J.: Evaluating the usability of visualization methods in an exploratory geovisualization environment, Int. J. Geographical Information Sci., 20(4), 425-448, 2006.

Lacassie, J. P., Roser, B., Ruiz del Solar, J., and Herve, F.: Discovering geochemical patterns using self-organizing neural networks: a new perspective for sedimentary provenance analysis, Sedimentary Geology, 165(1-2), 175-191, 2004.

Laga, P., Louwye, S., and Geets, S.: Paleogene and Neogene lithostratigraphic units (Belgium), Geologica Belgica, 4(1-2), 135152, 2001.

Lagrou, D., Dreesen, R., and Broothaers, L.: Comparative quantitative petrographical analysis of Cenozoic aquifer sands in Flanders (N Belgium): overall trends and quality assessment, Materials Characterization, 53, 317-326, 2004.

Lambrakis, N., Antonakos, A., and Panagopoulos, G.: The use of multicomponent statistical analysis in hydrogeological environmental research, Water Res., 38(7), 1862-1872, 2004.

Lischeid, G.: Taming awfully large data sets: using self- organizing maps for analyzing spatial and temporal trends of water quality data, Geophys. Res. Abstr., 5, 01879, 2003.

Love, D., Hallbauer, D., Amos, A., and Hranova, R.: Factor analysis as a tool in groundwater quality management: two southern African case studies, Phys. Chem. Earth, 29(15-18), 1135-1143, 2004.

Mercier, G., Hubert-Moy, L., Houet, T., and Gouéry, P.: Estimation and monitoring of bare soil/vegetation ratio with SPOT VEGETATION and HRVIR, IEEE Trans. Geosci. Rem. Sens., 43(2), 
348-354, 2005.

Mingoti, S. A. and Lima, J. O.: Comparing SOM neural network with Fuzzy c-means, K-means and traditional hierarchical clustering algorithms, European J. Operational Res., 174, 17421759, 2006.

Ozerdem, M. S., Ustundag, B., and Demirer, R. M.: Self-organized maps based neural networks for detection of possible earthquake precursory electric field patterns, Advances in Engineering Software, 37(4), 207-217, 2006.

Openshaw, S. and Turton, I.: A parallel Kohonen algorithm for the classification of large spatial datasets, Computers and Geosciences, 22(9), 1019-1026, 1996.

Penn, B. S.: Using self-organizing maps to visualize highdimensional data, Computers and Geosciences, 31(5), 531-544, 2005.

Poulton, M. M., Sternberg, B. K., and Glass, C. E.: Location of subsurface targets in geophysical data using neural networks, Geophys., 57(12), (1534-1544), 1992.

Richardson, A. J., Risien, C., and Shillington, F. A.: Using selforganizing maps to identify patterns in satellite imagery, Progress in Oceanography, 59(2-3), 223-239, 2003.
Sanchez-Martos, F., Aguilera, P. A., Garrido-Frenich, A., Torres, J. A., and Pulido-Bosch, A.: Assessment of groundwater quality by means of self-organizing maps: application in a semi-arid area, Environ. Manage., 30(5), 716-726, 2002.

Skupin, A. and Hagelman, R.: Attribute space visualization of demographic change, Eleventh ACM international symposium on Advances in geographic information systems, New Orleans, Louisiana, USA, 2003.

Takatsuka, M.: An application of the self-organizing map and interactive 3-D visualisation to geospatial data, GeoComputation'01 (6th International Conference on GeoComputation, Brisbane, Australia, 2001

Ultsch, A. and Herrmann, L.: The architecture of emergent selforganizing maps to reduce projection errors, in: ESANN2005 13th European Symposium on Artificial Neural Networks, Bruges, Belgium, 1-6, 2005.

Vesanto, J., Himberg, J., Alhoniemi, E., and Parhankangas, J.: Selforganizing map in Matlab: the SOM Toolbox, in: Matlab DSP Conference, Espoo, Finland, 35-40, 1999. 